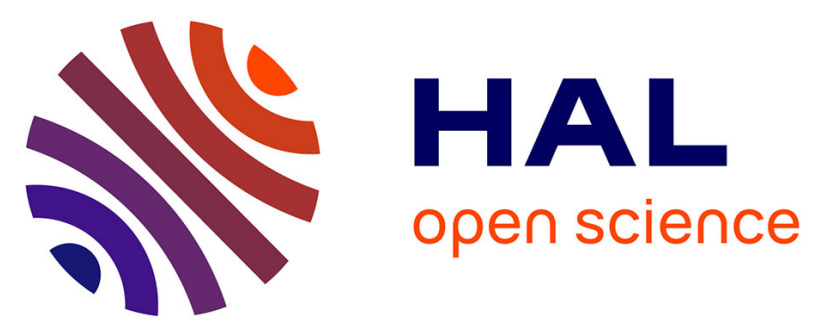

\title{
Relevance of a novel external dynamic distraction device for treating back pain.
}

\author{
Fahed Zaïri, Mélissa Moulart, Christian Fontaine, Fahmi Zaïri, Vincent \\ Tiffreau, Régis Logier
}

\section{- To cite this version:}

Fahed Zaïri, Mélissa Moulart, Christian Fontaine, Fahmi Zaïri, Vincent Tiffreau, et al.. Relevance of a novel external dynamic distraction device for treating back pain.. Proceedings of the Institution of Mechanical Engineers. Part H, Journal of engineering in medicine, In press, 10.1177/0954411920971401 . hal-03138340

\section{HAL Id: hal-03138340 \\ https://hal.univ-lille.fr/hal-03138340}

Submitted on 11 Feb 2021

HAL is a multi-disciplinary open access archive for the deposit and dissemination of scientific research documents, whether they are published or not. The documents may come from teaching and research institutions in France or abroad, or from public or private research centers.
L'archive ouverte pluridisciplinaire HAL, est destinée au dépôt et à la diffusion de documents scientifiques de niveau recherche, publiés ou non, émanant des établissements d'enseignement et de recherche français ou étrangers, des laboratoires publics ou privés. 
Zaïri F, Moulart M, Fontaine C, Zaïri F, Tiffreau V, Logier R.

Relevance of a novel external dynamic distraction device for treating back pain.

Proc Inst Mech Eng H. 2020 Nov 27:954411920971401.

doi: $10.1177 / 0954411920971401$.

PMID: 33243076

\section{Relevance of a novel external dynamic distraction device for treating back pain}

Fahed Zaïri $^{{ }^{*}}$, Mélissa Moulart ${ }^{2}$, Christian Fontaine $^{3}$, Fahmi Zaïri ${ }^{4}$, Vincent Tiffreau ${ }^{5}$, Régis

$$
\text { Logier }^{6}
$$

${ }^{1}$ Department of Neurosurgery, Hôpital Privé le Bois, Ramsay Général de Santé, 59000 Lille, France

${ }^{2}$ Japet Medical Device, 59000 Lille, France

${ }^{3}$ Department of Anatomy, Lille University Hospital, 59000 Lille, France

${ }^{4}$ Lille University, Civil Engineering and geo-Environmental Laboratory (EA 4515 LGCgE), 59000 Lille, France

${ }^{5}$ Rehabilitation Unit, Lille University Hospital, 59000 Lille, France

${ }^{6}$ CIC-IT, Lille University Hospital, 59000 Lille, France

*corresponding author 
fahed.zairi@gmail.com

Tel.: 0033666546169

Fax: 0033320446623

\section{Abstract}

Introduction: Low back pain is a common, expensive and disabling condition in industrialized countries. There is still no consensus for its ideal management. Believing in the beneficial effect of traction, we developed a novel external dynamic distraction device. The purpose of this work is to demonstrate that external distraction allows limiting the pressure exerted on orthostatism on the lower intervertebral discs.

Materials and methods: We firstly used the Anybody Modeling System, which is a validated musculoskeletal software. The device has been implemented in the model and we calculated the lower disc pressure while traction forces were applied by the four actuators. Secondly, we performed an anatomical study using a Biomet cadaver placed in a sitting position. The two belts of the device were tightened at the base of the thorax and on the waist, respectively. A pressure sensor was placed in the lower discs under fluoroscopic control through a Jamshidi needle. The intradiscal pressure was then measured continuously at rest while applying a traction force of $20 \mathrm{Kg}$.

Results: Both numerical and cadaveric studies have demonstrated a decrease in intradiscal pressures after application of a traction force by the external device. Using the numerical model, we have shown that tensile forces below $50 \mathrm{Kg}$ in total were sufficient. The application of superior forces is useless and potentially deleterious.

Conclusion: External dynamic distraction device is able to significantly decrease the intradiscal pressure in a sitting or standing position. The effects are obtained using "reasonable" traction forces, inferior to those applied by most conventional traction tables. However, the therapeutic effects need to be proven using clinical studies.

Keywords: Back pain; Actuators; Traction; Disc pressure; Anybody Modeling System

\section{Introduction}

Back pain is becoming an increasing concern in most industrialized countries. Indeed, it is estimated that $80 \%$ of the adult population suffers or will suffer from low back pain with an annual prevalence of $30 \%^{1,2}$. This frequency more than tripled in Europe and in the US between 1980 and $2000^{1,2}$. Chronic low back pain, whose evolution is by definition longer than 3 months, concerns only $20 \%$ of these patients, that is to say 10 to $20 \%$ of the general 
population with a preferred age ranged between 35 and 45 years. In industrialized countries, low back pain is a major public health problem because it generates significant costs in healthcare and is one of the leading causes of disability and sick leave ${ }^{3,4}$. From a physiopathological point of view, chronic low back pain is due to degenerative changes in the intervertebral disc and articular joints ${ }^{5,6}$. The lower segments are the more frequently and severely affected in clinical practice (L4L5 and L5S1 segments). Many risk factors have been highlighted such as smoking, overweight, sedentary lifestyle, and some occupations that require heavy loads ${ }^{7,8}$. The exaggerated mechanical stresses are clearly identified to accelerate the degeneration and thus aggravate the symptoms.

In spite of its frequency and its social impact, there is no consensus for the treatment of chronic low back pain ${ }^{9,10}$. The surgery failed to show its effectiveness in the absence of radicular pain, except in very particular situations ${ }^{11,12}$. In the same way, the majority of conservative treatments have not shown clear evidence of their effectiveness ${ }^{13}$. Among them, lumbar traction is a commonly used method to treat patients with low back pain with or without sciatica. In the UK and the US, lumbar traction is used by 41 and $77 \%$ of outpatient rehabilitation providers, respectively ${ }^{14,15}$. Thus, there is a discordance between the lack of evidence-based recommendation and how lumbar traction is regarded in current clinical practice, which is explained by the great heterogeneity of practices and the methodological problems found in most clinical studies ${ }^{16}$. Believing in the beneficial effect of traction, we developed an external distraction device. The purpose of this work is to demonstrate that external distraction allows to limit the pressure exerted on orthostatism on the lumbar spine and mainly on the intervertebral disc.

\section{Materials and Methods}

\subsection{Numerical assessment}

\section{a. "Anybody" model}

Anybody Modeling System (AMS) is a validated musculoskeletal software revolving around inverse dynamic simulations able to calculate individual muscle forces, joint contact forces and pressures ${ }^{17}$. Each body part is implemented using validated cadaveric or anatomical data ensuring high accuracy and anatomical fidelity of the model. Every bones, joints, muscles, ligaments and tendons are represented (Figure 1).

Finally, AMS allows the importation of computer assisted design (CAD) components in order to study their effects in interaction with the body. Note that anthropometric measures can be modified. 


\section{b. Exoskeleton model}

The aim of the exoskeleton is to apply vertical traction forces to reduce pressure on the lumbar spine in the upright position (Figure 2A). In order to preserve both proper spinal alignment and freedom of motion, the device is composed of two sets of actuators positioned on both side of the body. The traction is produced by these four actuators, each of them represented by a pivot joint between the upper part and the lower part of the device (Figure 2B). These joints are activated by electric motors thanks to worm screws. At the end of each of them, there is a ball joint to preserve the motion of the trunk. Each actuator can generate a force of $8 \mathrm{Kg}$, ensuring a maximum distraction force of $32 \mathrm{Kg}$.

\section{c. Implementation of the exoskeleton in Anybody}

As the simulation does not include the skin, we used the torso on which the exoskeleton was designed to position the device in the simulation. The torso used for the design of the prototype is a standard morphology transmitted by a local orthoprosthesist. Then, a skeleton has been fitted inside the CAD of the body with size adaptation relative to the body dimension. The skeleton was obtained from a free CAD on Grabcad (Figure 3). The skeleton inside the CAD was then fitted to the Anybody Model to position the torso, therefore fitting the position of the exoskeleton in the simulation. Finally, the connection points were visually fitted to the solid part of the pelvis and the thorax.

Therefore, it was necessary to simplify the model (Figure 2C). First, a perfect model was applied for the actuators (no friction) to simplify the calculations. Secondly, as the AMS software performs the simulations by inverse dynamics operations, we had to constrain the DOF. The top ball joint has been replaced by a universal joint to avoid rotation in the actuators along the $\mathrm{z}$ axis. Similarly, the pivot joint between the two parts of each actuator has been replaced by a slide joint to limit rotation. Finally, the connection between the body and the belts has been simplified as a housing to prevent movement

\section{d. Study protocol}

For this study, we have chosen morphometric data to get closer to the European average for a man. We parameterized a height of 1.76 meters, a weight of 74 kilograms and a lumbar disc area of $19.8 \mathrm{~cm}^{2}$. The intradiscal pressure $(\mathrm{P})$ was estimated by dividing the force $(\mathrm{F})$ with the 
corresponding disc area applying a correction factor. The correction factor considered the non-uniform load distribution in the disc. In accordance with the Nachemson study ${ }^{18,19}$ as confirmed by Brinckmann et al. ${ }^{20}$ and Cripton et al. ${ }^{21}$, we applied a correction factor of 0.66. Thus, the final formula to calculate the intradiscal pressure was $\mathrm{P}=\mathrm{F} /(\mathrm{S} \times 0.66)$. Even if the actuators of our device can not develop more than $8 \mathrm{~kg}$ of traction force, we decided to realize extreme simulations of 0 to $100 \mathrm{Kg}(25 \mathrm{Kg}$ per actuator) in order to define the optimal traction force and to analyze changes of disc pressure during extreme traction.The measurements were performed for the two lower discs L4L5 L5S1, which are most frequently affected in clinical practice. We also decided to analyze the activity of the lumbar muscles to detect and measure any possible reaction contractile activity.

\subsection{Anatomical study}

\section{a. Cadaver characteristics}

The full body of a 62-year-old woman (height $1.62 \mathrm{~cm}$, weight $70 \mathrm{Kg}$ ) was used for the study. The cadaver has been prepared using the Biomet liquid. She had no history of spinal surgery. Radiological evaluation confirmed a disc high superior to $10 \mathrm{~mm}$ from L3 to S1. Similarly, there was nosclerosis of the endplates or voluminous osteophytes, overall confirming the absence of severe disc degeneration at those levels.

\section{b. Measurement technique}

A table of maintenance has been made for this study. The corpse is kept in a sitting position, in rectitude avoiding any support under the arms, likely to reduce the weight of the body. Due to the elasticity of tissues, we waited thirty minutes before starting any measurement to reach the plateau phase. A Jamshidi needle has been inserted percutaneously through a strict midline posterior approach. The placement has been performed under a strict anteroposterior and lateral fluoroscopic control (Figure 4). On the lateral radiograph, the disc was divided into three zones: posterior, medial and anterior (Figure 4C). The needle was placed respectively in the three zones beginning with the posterior, then median and finally anterior. Due to the bony overlapping of the iliac wing, it was difficult to certify the proper positioning of the needle at L5S1 level and measurements were thus made on the L3L4 and L4L5 discs. The device consists of two belts that were tightened at the base of the thorax and on the waist (over the iliac crests), respectively; no rigid attachment to the cadaver was performed, to maintain the potential effects of slips that could be encountered in therapeutic condition. 


\section{c. Measurement protocol}

After having placed the needle, a pressure sensor was inserted through. We used a Gaeltec sensor coupled to the Picolog analysis software. Once the sensor introduced, awaiting time of 5 minutes was respected in order to reach an equilibrium, because of the minimal tissue lesions induced by the puncture. We then performed five measurements for each zone and for each disc. For each measurement the same protocol has been respected. After recording the base pressure, we applied a force of $5 \mathrm{~kg}$ per actuator (total of $20 \mathrm{~kg}$ ) for 2 minutes. We then recorded the baseline pressure for 5 minutes to regain balance before starting a new measurement. We then performed a prolonged recording to measure the evolution of the disc pressure when a prolonged distraction is applied. We placed the sensor in the middle of the disc and after observing a latency of 5 minutes, we measured the disc pressure while a force of $5 \mathrm{Kg}$ per actuator for 30 minutes was applied.

\section{Results}

\subsection{Numerical assessment: AMS}

\section{a. Development of the intradiscal pressure}

For the L4L5 disc, it was found that the disc pressure gradually decreased from 0.41 to 0.2 $\mathrm{MPa}$ for a total traction force close to $50 \mathrm{Kg}$. By increasing the tensile force, paradoxically, a rise in the disc pressure was observed (Figure 5A).

For the L5S1 disc, the intradiscal pressure decreased from 0.42 to $0.17 \mathrm{MPa}$ for a total traction force close to $60 \mathrm{Kg}$. Similarly, by increasing the traction, we observed a rise in the discal pressure that reached $0.2 \mathrm{MPa}$ for $100 \mathrm{Kg}$ of traction (Figure 5B).

Under the conditions of this simulation, the optimum total traction force seems to be close to $50 \mathrm{~kg}$. In addition to safety concerns, a higher traction force seems useless or even deleterious.

\section{b. Muscles activity}

At the lumbar level, we have three powerful and stabilizing muscles that are inside and outside, the multifidus, the longissimus and the iliocostal, acting in compression ${ }^{22}$. The activity of these muscles was recorded in parallel with the disc pressure (Figure 6). We found that there was little muscle activity for traction forces below $40 \mathrm{Kg}$. Beyond $50 \mathrm{Kg}$, we 
observed a significant and progressive increase in the activity of these three muscles, exceeding twice the basis activity for the longissimus and three times the basis activity for both multifidus and iliocostal.

\subsection{Anatomical study}

As stated previously, the measurements were performed on L3L4 and L4L5 discs, as L5S1 disc was not easily identifiable on the fluoroscopic control, because of bony overlapping of the iliac wing, which did not allow confirming the proper positioning of the sensor with certainty.

For the L3L4 disc, we measured a significant decrease in intradiscal pressure during the distraction phase which remained stable. This decrease was reproducible in the five completed registrations. Standardized results are shown in Figure 7. We found that the decrease in pressure was greater in the middle and at the back of the disc, whereas it was less significant at the front of the disc. Indeed, the pressure drop reaches $43.96 \%$ in the middle, $27.82 \%$ in the back and only $17.90 \%$ in the front of the disc. For the L4L5 disc, we also measured a significant drop in pressure after activation of the actuators. This decrease was as stable and reproducible in the five recordings made. We found that the pressure drop was more significant at the back and the middle of the disc while it was minimal at the front of the disc. The normalized averages obtained for each zone of each disc are shown in Figure 8.

We also found that the pressure drop obtained under the effect of traction was durable over time. Indeed, the decrease in pressure recorded in the middle of the L3L4 disc remained significant (up to $40 \%$ ) beyond thirty minutes of continuous traction (Figure 9).

\section{Discussion}

Low back pain is a common, expensive and disabling condition in industrialized countries ${ }^{1-4}$. The pathophysiology is complex but the exaggerated mechanical stresses were clearly identified as a main pejorative factor. The existing therapeutic solutions are multiple but to date the prognosis is still often unfavourable, reflecting the need for new therapeutic tools ${ }^{23}$. Among the existing solutions, traction is very popular but no study has been able to demonstrate its clinical effectiveness in the medium or long term ${ }^{16}$. However, experimental studies have shown that traction tables are likely to increase the height of the intervertebral disc and even reduce the conflicts between the disc and the nerve roots in case of associated sciatica $^{24-27}$. To date, the lack of evidence of effectiveness is likely to be related to a lack of technical solution, rather than a lack of concept. In this perspective, we developed an 
exoskeleton to obtain a distraction in orthostatism. Thus, the traction can be applied more prolonged, on a subject in a position of function and potentially in motion and in activity insofar as the actuators allow the maintenance of the amplitudes of movement. The objective would be to reduce the mechanical stress exerted on the lumbar disc (L4L5 and L5S1 being the most frequently affected), while maintaining the activity of the patient (recreation or professional). It would also aim to limit the muscular deconditioning caused by the inactivity or rigid contention belts sometimes proposed.

In our study we demonstrated that an external distraction was able to induce a significant decrease in intradiscal pressure. Using the numerical model, we have shown that tensile forces below $50 \mathrm{Kg}$ in total were sufficient. The application of superior forces is useless because it is accompanied by a deleterious increase of the disc pressure. This effect can be explained in large part by the reflex muscular activity (recorded in our study) but also by the elastic properties of the ligament and tendinous structures not represented in the anybody model. Indeed, the developers stated: "when building the lumbar spine model the original idea was to include ligaments as well. But at some point, we decided not to include the ligaments in the model, because of lack of readily available information about the mechanical properties and slack lengths. We were in fear that ligaments with wrong properties might give worse results than excluding them". Note that most traction tables currently apply forces up to $100 \mathrm{Kg}$ in clinical practice 27,28 . The absence of representation of the ligaments is not the only limit of this model. The skin is also not represented in AMS, and the device had to be attached to the skeleton. As a result, the sliding of the device on the skin and the soft tissues is not taken into account, which may increase the physiological effects observed. In addition, the disc pressure is not directly measured but calculated according to the defined surface and by means of a chosen correction factor, which can be a source of approximation. The biomechanics of the soft tissue should be considered in further more accurate models ${ }^{30,31}$. In the current study, it was therefore necessary to carry out direct measurements "in vivo" in order to limit these biases.

Unlike cadavers prepared with formaldehyde, we used a BIOMET cadaver, in order to preserve much of the elasticity of the tissues. This cadaveric study has demonstrated that the application of external traction significantly reduces disc pressure. This pressure drop is significant, reproducible and durable over time, as demonstrated during the prolonged recording of thirty minutes, allowing to appreciate the potential therapeutic effects. Note that 
we measured larger effects in the front and middle of the disc while the pressure drop was lower at the front of the disc at the L3L4 and L4L5 levels. This is probably related to a postural effect. Indeed, the corpse was sitting slightly leaning forward, which can induce a slight inversion of curvature (lower lordosis) and increase the stresses exerted on the front of the $\operatorname{disc}^{27,28}$.

This cadaveric study has certain limitations. First, muscle activity is non-existent and its effect cannot be measured. In addition, it would have been useful to perform measurements on several subjects to confirm reproducibility. However, the combination of a simulation on a validated model and a cadaveric study with direct measurements, makes it possible to validate the effect of external distraction on the decrease in intradiscal pressure. The therapeutic effect of this device deserves to be carefully studied, and for this an observational clinical study is currently ongoing.

\section{Conclusion}

In summary, external dynamic distraction device is able to significantly decrease the intradiscal pressure in a sitting or standing position. The effects are obtained using "reasonable" traction forces, inferior to those applied by most conventional traction tables. However, the therapeutic effects need to be proven using clinical studies.

\section{Conflict of interests}

None declared.

\section{Acknowledgments}

The authors would like to thank the Clinical Anatomy Laboratory for providing the cadaver. The authors are grateful to Maurice Demeulaere, Fabien Descamps, Antoine Vanholber and Franck Stevendart for their assistance during the experiments.

\section{References}

1. Andersson, G.B., 1999. Epidomiological features of chronic low-back pain. Lancet 354, 581-585.

2. Jayson, M.I.V., 1996. ABC of work related disorders: Back pain. BMJ 313, 355-358.

3. Maher, C., Underwood, M., Buchbinder, R., 2017. Non-specific low back pain. Lancet 389, 736-747. 
4. Hartvigsen, J., Hancock, M.J., Kongsted, A., Louw, Q., Ferreira, M.L., Genevay, S., et al. 2018. What low back pain is and why we need to pay attention. Lancet 391, 2356-2367.

5. Borenstein, D., 2013. Mechanical low back pain: A rheumatologist's view. Nature Reviews Rheumatology 9, 643-653.

6. Bogduk, N., 2009. On the definitions and physiology of back pain, referred pain, and radicular pain. Pain 147, 17-19.

7. Taylor, J.B., Goode, A.P., George, S.Z., Cook, C.E., 2014. Incidence and risk factors for first-time incident low back pain: A systematic review and meta-analysis. The Spine Journal 14, 2299-2319.

8. Wippert, P.M., Fliesser, M., Krause, M., 2017. Risk and protective factors in the clinical rehabilitation of chronic back pain. Journal of Pain Research 10, 1569-1579.

9. Foster, N.E., Anema, J.R., Cherkin, D., Chou, R., Cohen, S.P., Gross, D.P., et al., 2018. Prevention and treatment of low back pain: Evidence, challenges, and promising directions. Lancet 391, 2368-2383.

10. Buchbinder, R., van Tulder, M., Oberg, B., Costa, L.M., Woolf, A., Schoene, M., et al., 2018. Low back pain: A call for action. Lancet 391, 2384-2388.

11. Copeland, B., 2007. Surgical versus nonsurgical treatment for back pain. The New England Journal of Medicine 357, 1255-1256.

12. Gaudin, D., Krafcik, B.M., Mansour, T.R., Alnemari, A., 2017. Considerations in spinal fusion surgery for chronic lumbar pain: Psychosocial factors, rating scales, and perioperative patient education - A review of the literature. World Neurosurgery 98, 21-27.

13. O'Connell, N.E., Cook, C.E., Wand, B.M., Ward, S.P., 2016. Clinical guidelines for low back pain: A critical review of consensus and inconsistencies across three major guidelines. Best Practice \& Research: Clinical Rheumatology 30, 968-980.

14. Thackeray, A., Fritz, J.M., Childs, J.D., Brennan, G.P., 2016. The effectiveness of mechanical traction among subgroups of patients with low back pain and leg pain: A randomized trial. Journal of Orthopaedic \& Sports Physical Therapy 46, 144-154.

15. Oh, H., Choi, S., Lee, S., Choi, J., Lee, K., 2018. The impact of manual spinal traction therapy on the pain and Oswestry disability index of patients with chronic back pain. Journal of Physical Therapy Science 30, 1455-1457.

16. Wegner, I., Widyahening, I.S., van Tulder, M.W., Blomberg, S.E., de Vet, H.C., Bronfort, G., et al., 2013. Traction for low-back pain with or without sciatica. Cochrane Database of Systematic Reviews 19, CD003010. 
17. Bassani, T., Stucovitz, E., Qian, Z., Briguglio, M., Galbusera, F., 2017. Validation of the AnyBody full body musculoskeletal model in computing lumbar spine loads at L4L5 level. Journal of Biomechanics 58, 89-96.

18. Nachemson, A., 1960. Lumbar intradiscal pressure. Experimental studies on post-mortem material. Acta Orthopaedica Scandinavica 43, 1-104.

19. Nachemson, A., 1966. Mechanical stresses on lumbar disks. Current Practice Orthopaedic Surgery $3,208-224$.

20. Brinckmann, P., Grootenboer, H., 1991. Change of disc height, radial disc bulge, and intradiscal pressure from discectomy. An in vitro investigation on human lumbar discs. Spine $16,641-646$.

21. Cripton, P.A., Bruehlmann, S.B., Orr, T.E., Oxland, T.R., Nolte, L.P., 2000. In vitro axial preload application during spine flexibility testing: Towards reduced apparatus-related artefacts. Journal of Biomechanics 33, 1559-1568.

22. Park, M.S., Moon, S.H., Kim, T.H., Oh, J., Lee, S.J., Chang, H.G., Shin, J.H., 2018. Paraspinal muscles of patients with lumbar diseases. Journal of Neurological Surgery. Part A Central European Neurosurgery 79, 323-329.

23. Moseley, G.L., 2017. Innovative treatments for back pain. Pain 158, S2-S10.

24. Chow, D.H.K., Yuen, E.M.K., Xiao, L., Leung, M.C.P., 2017. Mechanical effects of traction on lumbar intervertebral discs: A magnetic resonance imaging study. Musculoskeletal Science and Practice 29, 78-83.

25. Kuo, Y.W., Hsu, Y.C., Chuang, I.T., Chao, P.H., Wang, J.L., 2014. Spinal traction promotes molecular transportation in a simulated degenerative intervertebral disc model. Spine 39, E550-E556.

26. Chung, T.S., Yang, H.E., Ahn, S.J., Park, J.H., 2015. Herniated lumbar disks: Real-time MR imaging evaluation during continuous traction. Radiology 275, 755-762.

27. Tadano, S., Tanabe, H., Arai, S., Fujino, K., Doi, T., Akai, M., 2019. Lumbar mechanical traction: A biomechanical assessment of change at the lumbar spine. BMC Musculoskeletal Disorders 20,155-167.

28. Farajpour, H., Jamshidi, N., 2017. Effects of different angles of the traction table on lumbar spine ligaments: A finite element study. Clinics in Orthopedic Surgery 9, 480-488.

29. Nachemson, A., 1966. The load on lumbar disks in different positions of the body. Clinical Orthopaedics and Related Research 45, 107-122. 
30. Derrouiche, A., Zaouali, A., Zaïri, F., Ismail, J., Chaabane, M., Qu, Z., Zaïri, F., 2019. Osmo-inelastic response of the intervertebral disc. Proceedings of the Institution of Mechanical Engineers. Part H: Journal of Engineering in Medicine 233, 332-341.

31. Derrouiche, A., Zaïri, F., Zaïri, F., 2019. A chemo-mechanical model for osmo-inelastic effects in the annulus fibrosus. Biomechanics and Modeling in Mechanobiology, in press.

\section{Legends}

Figure 1: Anybody Modeling System default model.

Figure 2: Conceptual design of the device (A). The exoskeleton is composed of two belts tightened at the base of the thorax and on the waist (over the iliac crests), respectively, and linked by four actuators able to apply a traction force of $8 \mathrm{Kg}$ each. The kinematic of the exoskeleton is schematized (B). A simplified kinematic of the exoskeleton has been implemented (C).

Figure 3: Implementation of the exoskeleton in Anybody.

Figure 4: Anatomical protocol. The corpse is placed in a sitting position under strict fluoroscopic control (A). The Jamshidi needle is inserted percutaneously under anteroposterior and lateral fluoroscopy (B). On the lateral view (C) the disc is divided into three zone (posterior, middle, anterior). On the AP view, we ensure the strictly median placement (D).

Figure 5: Evolution of the intradiscal pressure in L4L5 (A) and L5S1 (B) as a function of the total traction force applied.

Figure 6: Evolution of the activity of the three main erector muscles of the back as a function of the total traction force applied.

Figure 7: Evolution of the intradiscal pressure in each zone of the L3L4 disc. Standardized results. 
Figure 8: Evolution of the intradiscal pressure (average of the five measurements) for each zone of the L3L4 (A) and L4L5 discs (B).

Figure 9: Prolonged recording in the middle of the L3L4 disc. There is a prolonged and significant decrease in pressure when traction is maintained.

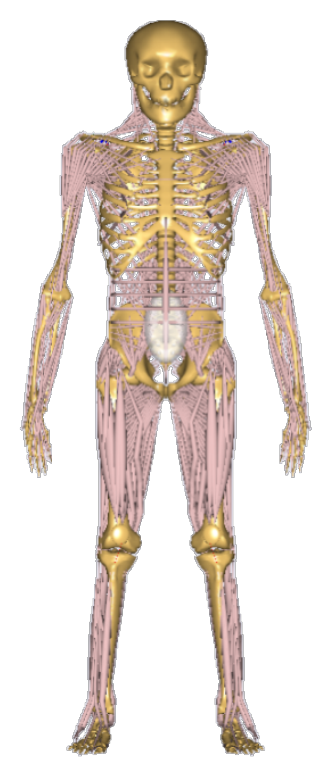

Figure 1: Anybody Modeling System default model 

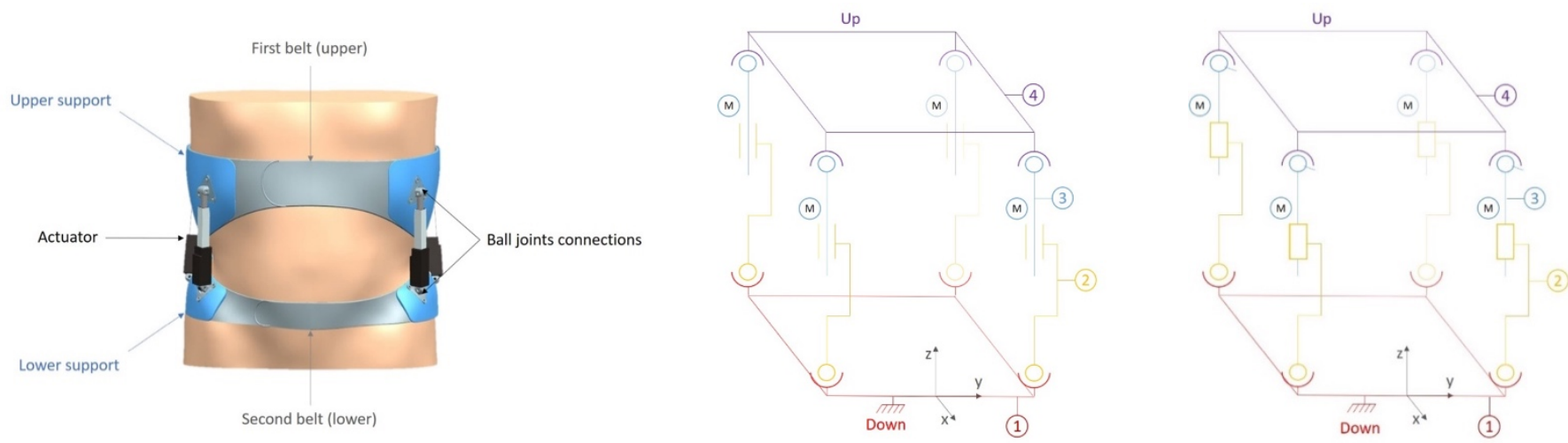

A

$\mathrm{B}$

$\mathrm{C}$

Figure 2: Conceptual design of the device (A). The exoskeleton is composed of two belts tightened at the base of the thorax and on the waist (over the iliac crests), respectively, and linked by four actuators able to apply a traction force of $8 \mathrm{Kg}$ each. The kinematic of the exoskeleton is schematized (B). A simplified kinematic of the exoskeleton has been implemented (C). 

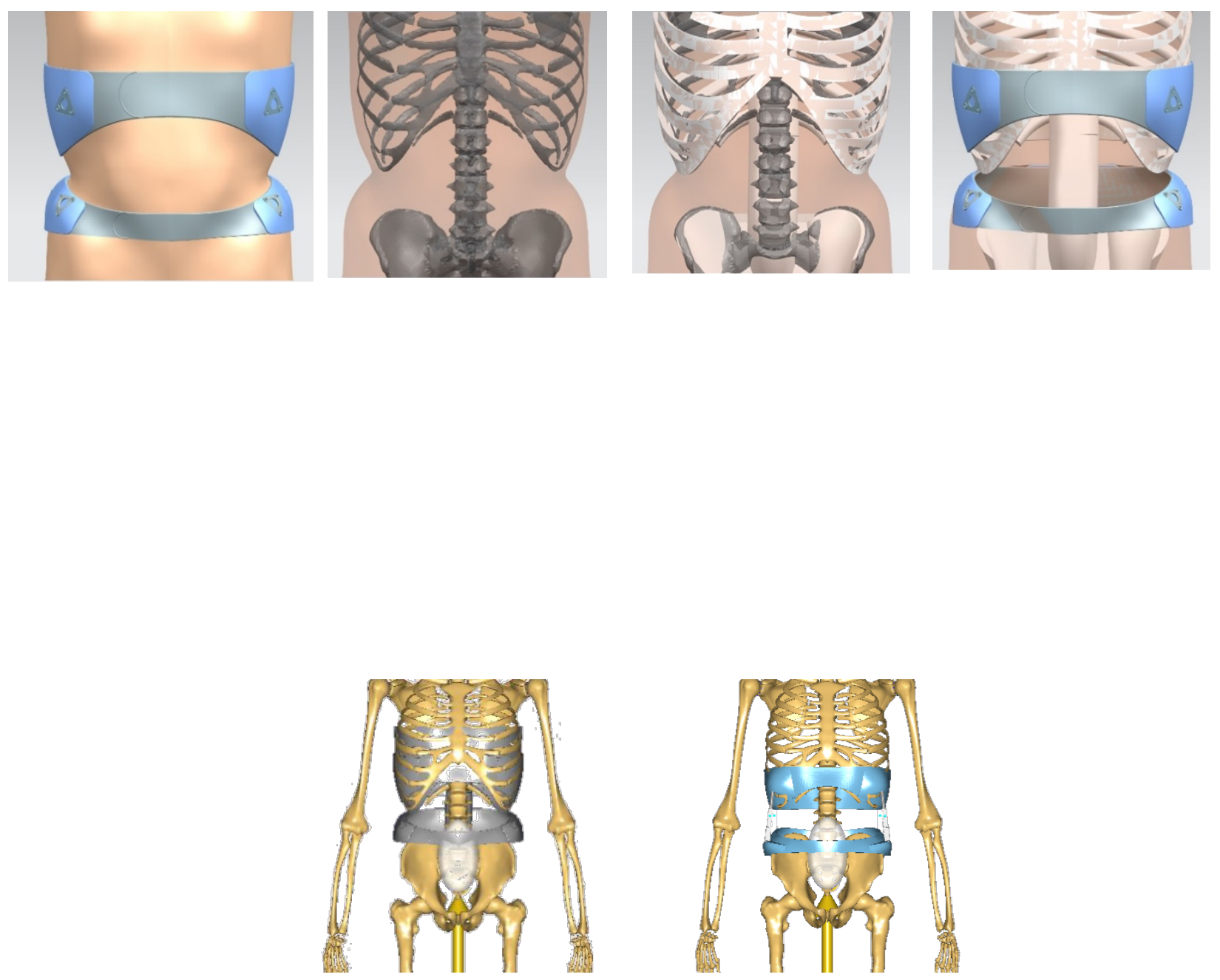

Figure 3: Implementation of the exoskeleton in Anybody.

Different steps: As the simulation does not include the skin, we used the torso on which the exoskeleton was designed to position the device in the simulation. Then, a skeleton has been fitted inside the CAD of the body with size adaptation relative to the body dimension. The skeleton inside the CAD was then fitted to the Anybody Model to position the torso, therefore 
fitting the position of the exoskeleton

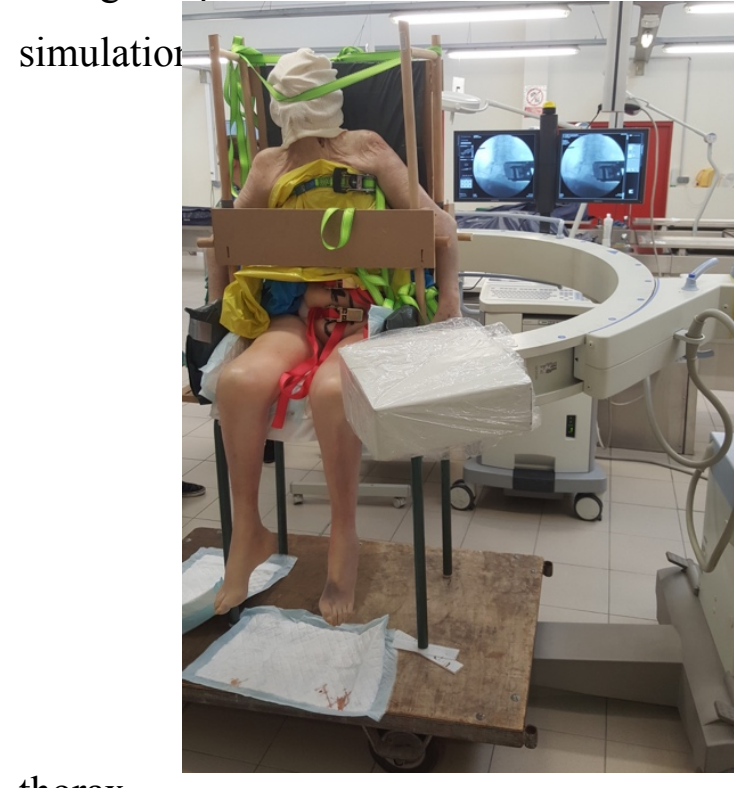

thorax.

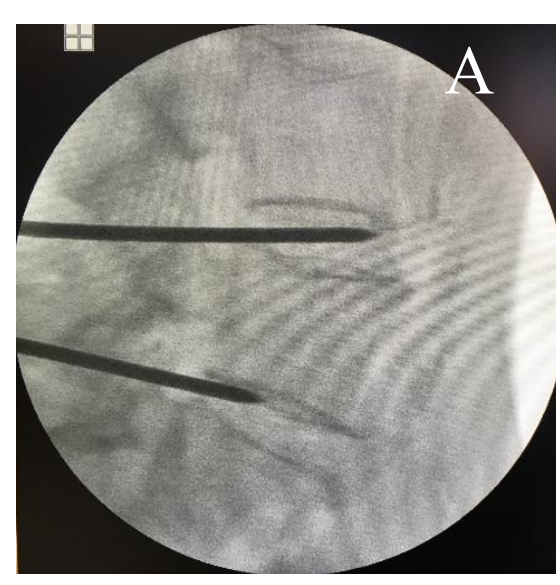

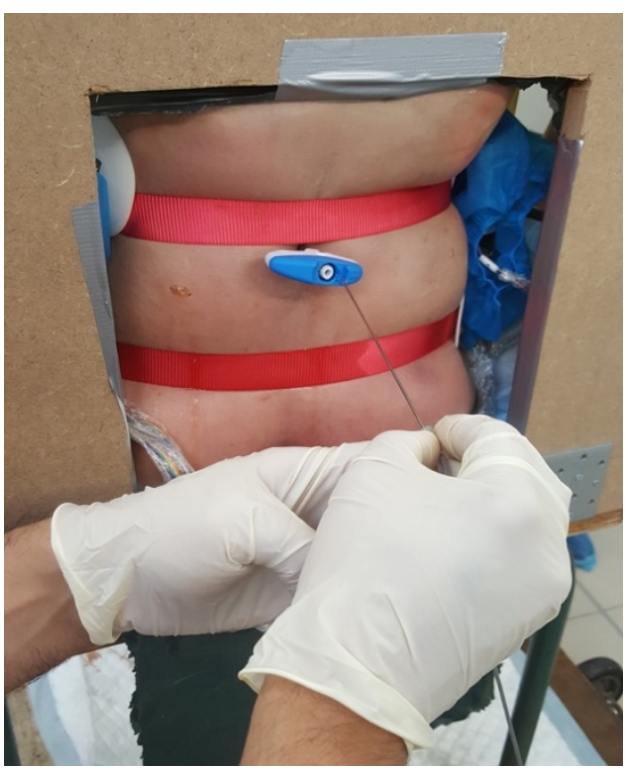

in the

points

were

visuall

y fitted

to the

solid

part of

the

pelvis

and the
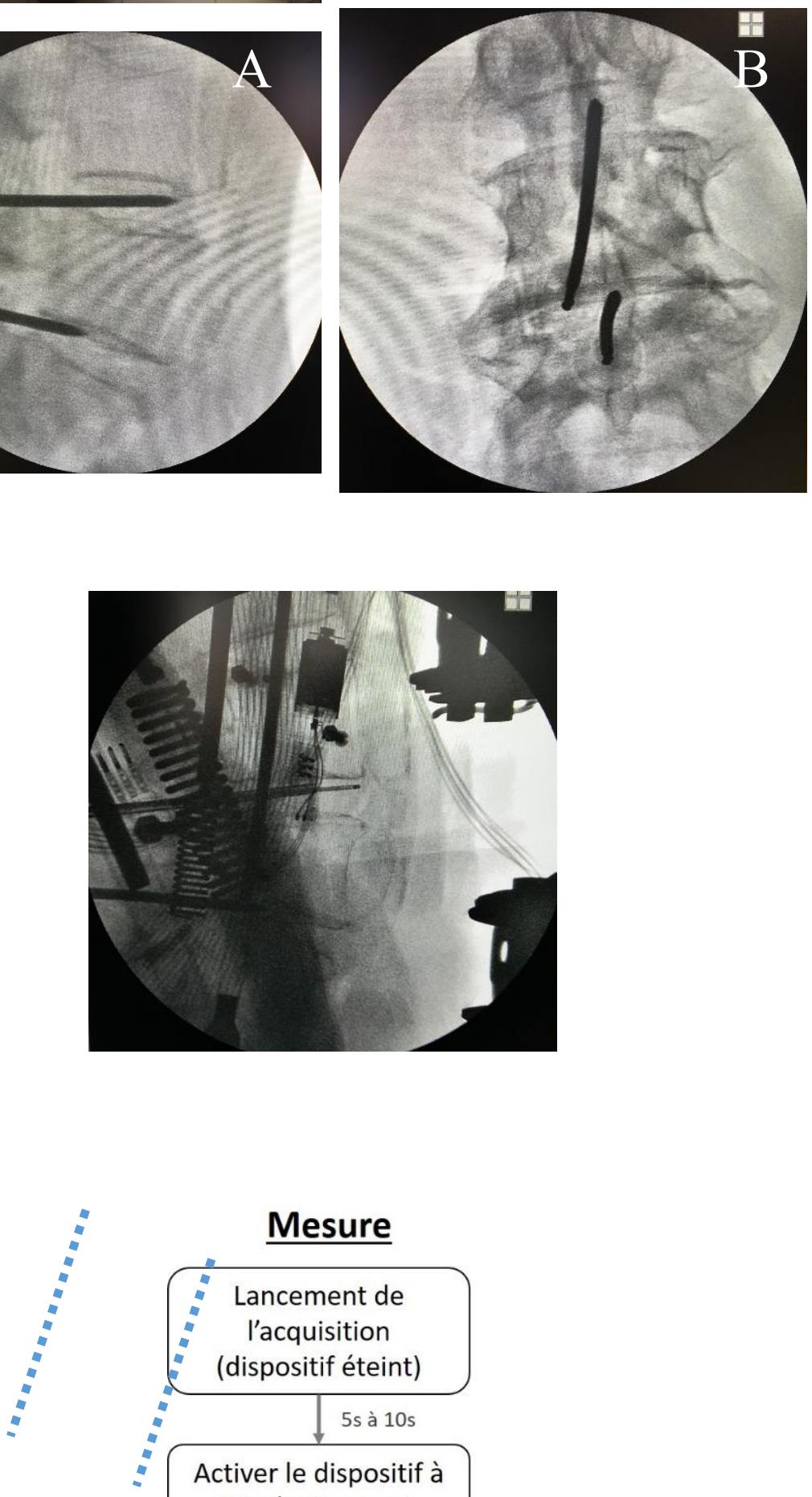


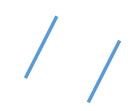

Figure 4: Anatomical protocol. The corpse is placed in a sitting position under strict fluoroscopic control (A). The Jamshidi needle is inserted percutaneously under anteroposterior and lateral fluoroscopy (B). On the lateral view (C) the disc is divided into three zone (posterior, middle, anterior). On the AP view, we ensure the strictly median placement (D).

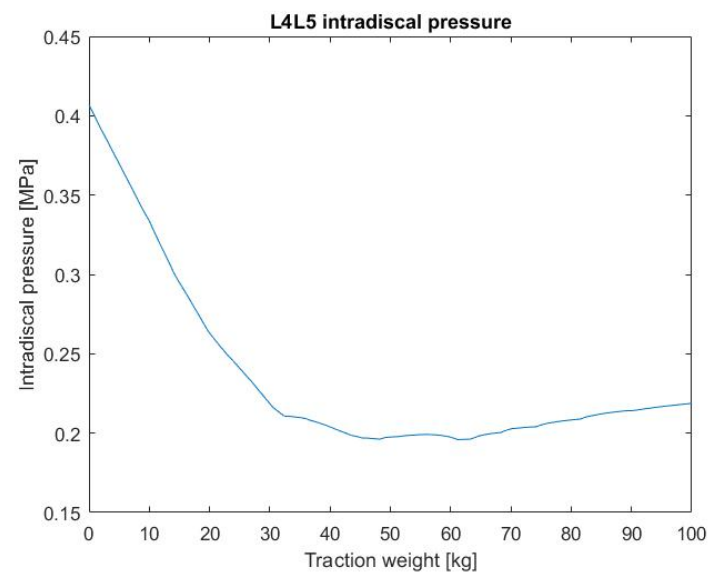


Figure 5: Evolution of the intradiscal pressure in L4L5 (A) and L5S1 (B) as a function of the total traction force applied.

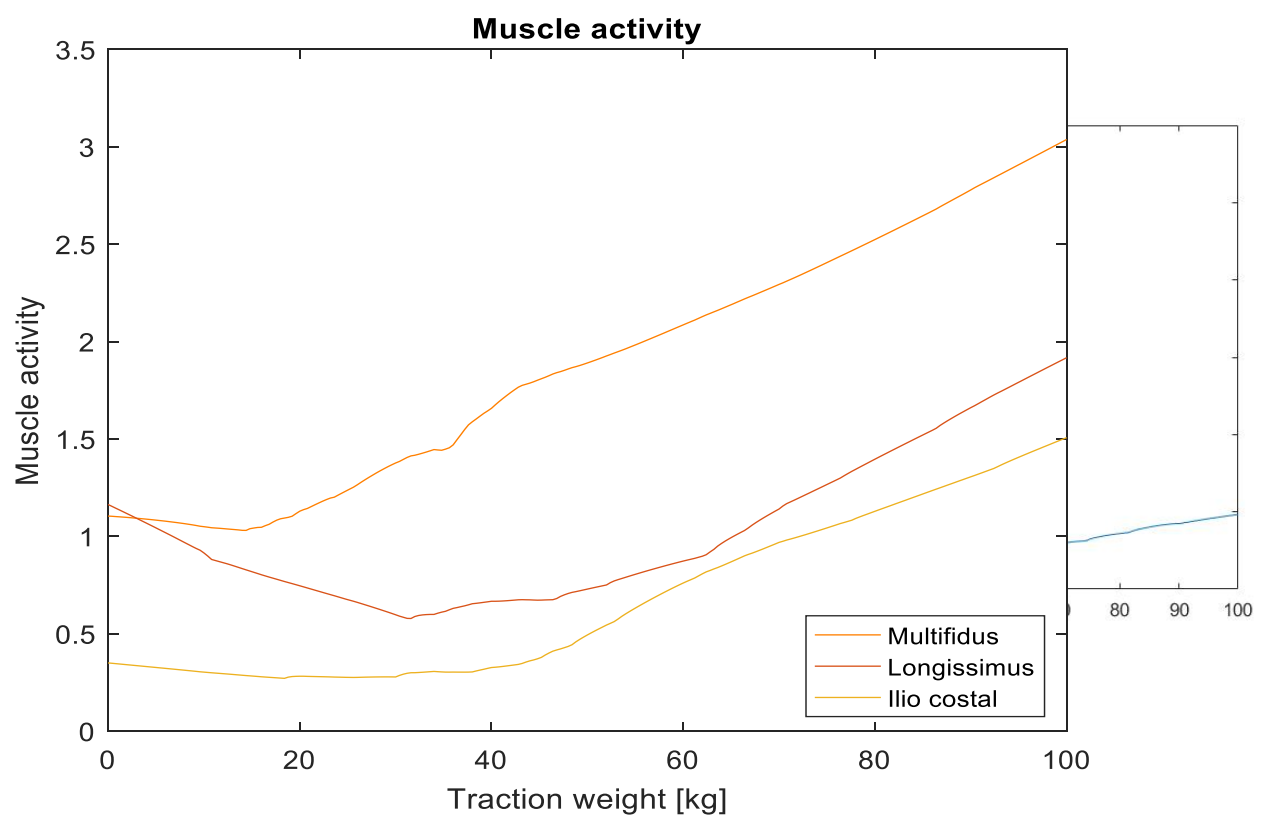


Figure 6: Evolution of the activity of the three main erector muscles of the back as a function of the total traction force applied.
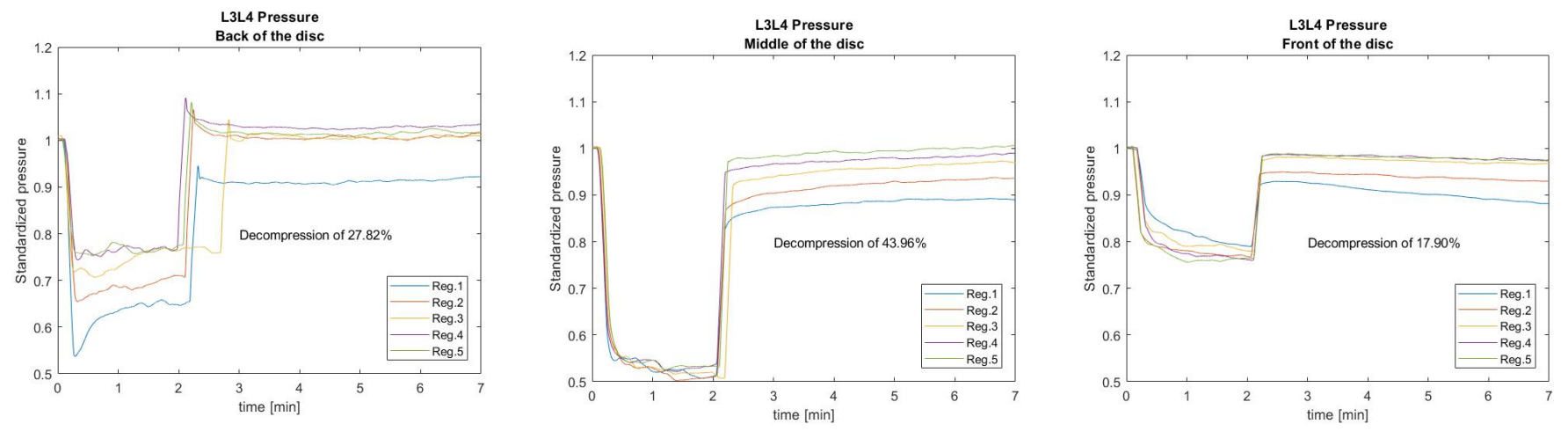
Figure 7: Evolution of the intradiscal pressure in each zone of the L3L4 disc. Standardized results.

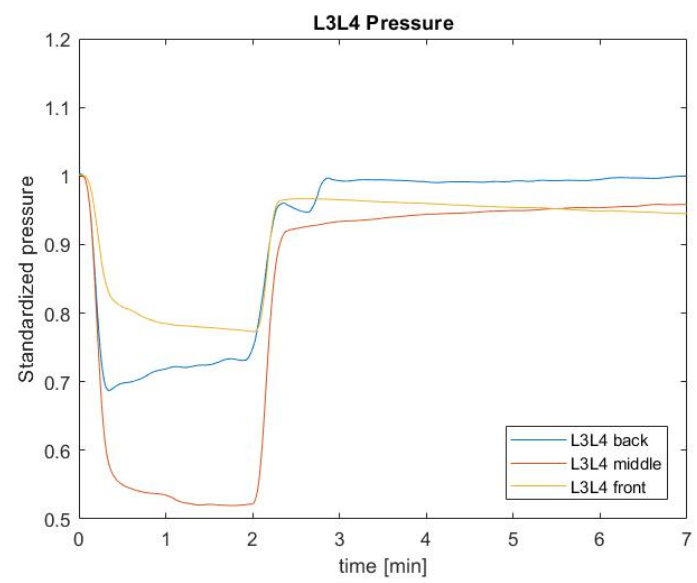


A

B

Figure 8: Evolution of the intradiscal pressure (average of the five measurements) for each zone of the L3L4 (A) and L4L5 discs (B).

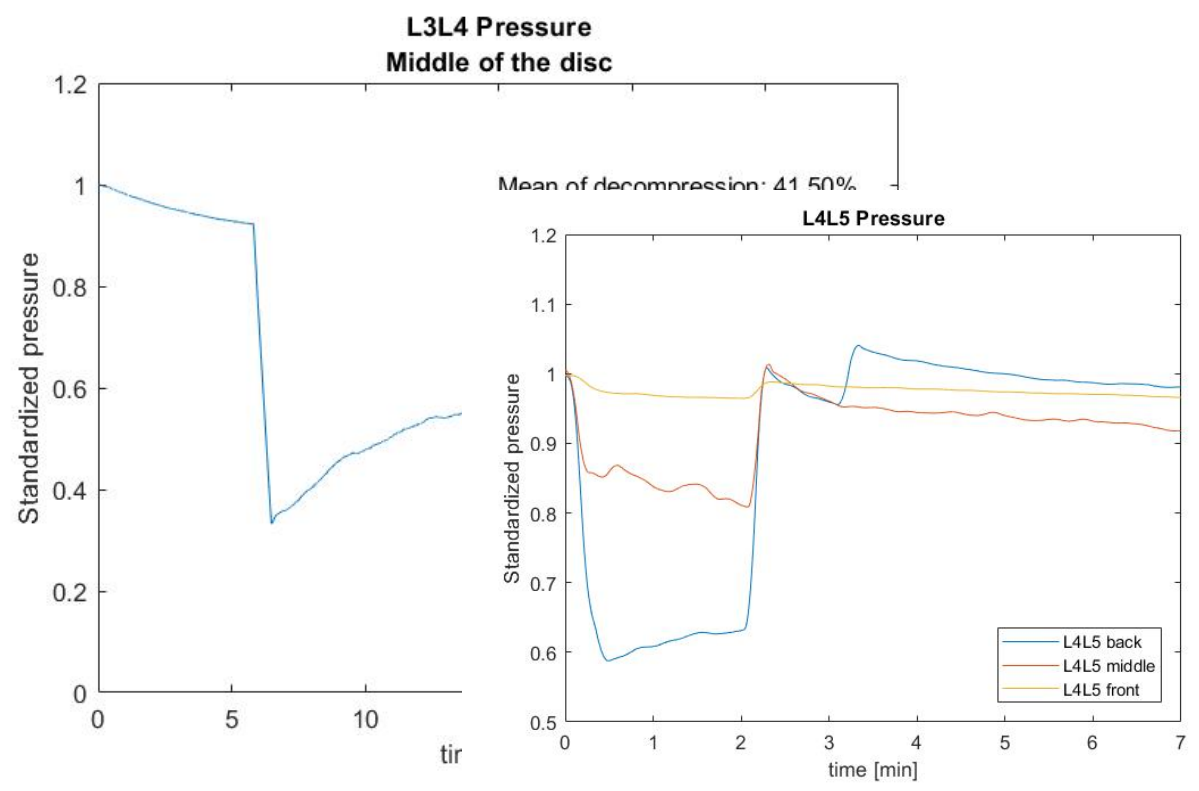


Figure 9: Prolonged recording in the middle of the L3L4 disc. There is a prolonged and significant decrease in pressure when traction is maintained. 